\title{
A Collaborative Unified Computing Platform for Building Information Modelling (BIM)
}

\author{
Steven Arthur, Dr Haijiang Li and Professor R Lark \\ School of Engineering, \\ Cardiff University, Queen's Buildings, 14-17 The Parade, Cardiff CF24 3AA, UK \\ \{arthurs, lih, lark\} @cardiff.ac.uk
}

\begin{abstract}
The current dominant computing mode in the AEC (Architecture, Engineering and Construction) domain is standalone based, causing fragmentation and fundamental interoperability problems. This makes the collaboration required to deal with the interconnected and complex tasks associated with a sustainable and resilient built environment extremely difficult.
\end{abstract}

This article aims to discuss how the latest computing technologies can be leveraged for the AEC domain and Building Information Modelling (BIM) in particular. These technologies include Cloud Computing, the Internet of Things and Big Data Analytics.

The data rich BIM domain will be analysed to identify relevant characteristics, opportunities and the likely challenges. A clear case will be established detailing why BIM needs these technologies and how they can be brought together to bring about a paradigm shift in the industry.

Having identified the potential application of new technologies, a future platform will be proposed. It will carry out large scale, realtime processing of data from all stakeholders. The platform will facilitate the collaborative interpretation, manipulation and analysis of data for the whole lifecycle of building projects. It will be flexible, intelligent and able to autonomously execute analysis and choose the relevant tools. This will form a base for a step-change for computing tools in the AEC domain.

Keywords: Big Data, Collaboration, Cloud Computing, Building Information Modelling, IoT, BIM

\section{Introduction}

The AEC industry has been slow to adapt to technological change, resulting in stagnation or even decline over the last 40 years [1]. Fragmentation, competition, a deeply embedded conservative approach [2] and other factors have slowed the adoption of new technology but the situation is slowly 
beginning to change. The most significant development has been the adoption of BIM, a digital representation of the physical and functional characteristics of buildings or infrastructure. The full potential of BIM is far from being fully exploited. This article proposes a BIM Platform to bring together data from multiple sources and contemporary technologies to achieve BIM's potential through collaboration and the full use of all data.

The current situation results in untapped insights from rich data sources and unrealised collaboration opportunities. Stakeholders work individually on their part of the project with little or no collaboration with other parties. Data is either not collected from potential sources or the expertise is not available to make use of it.

New technologies can unlock the potential of BIM. The Internet of Things provides a rich source of new data which can be analysed using Big Data Analytics. Cloud Computing enables real-time collaboration, high availability and access to scalable resources. This article proposes a BIM Platform which unifies these technologies and brings myriad benefits to stakeholders operating as a truly collaborative network.

This article is organised as follows: Sect. 2 reviews the factors leading to the need for a Collaborative Unified Computing Platform for BIM. Sect. 3 describes the methods for bringing about a computing paradigm shift for BIM. Sect. 4 explains the components, architecture, methodology and implementation of the BIM platform. Sect. 5 discusses specific uses of the BIM Platform. The article ends with a conclusion in Sect. 6.

\section{The Need for a Collaborative Unified Computing Platform for BIM}

Many industries have been changed, created or have even disappeared because of disruptive technology. Travel, journalism, television, music, advertising and many more have been fundamentally changed. The history and status quo of the AEC domain is very different.

\subsection{Technological Inertia in the AEC Domain}

There are many reasons for technological inertia in the AEC domain. There is a great amount of fragmentation and a large job could involve dozens of subcontractors, architectural and engineering firms, managers, etc. Furthermore, the parties involved vary from project to project which makes it difficult to synchronise activities or develop collaborative systems which persist. Competition in the industry creates a disincentive to invest in new technology when working on any one project despite long-term advantages. Other factors include concerns about the benefits being too small to justify the initial costs and a conservative approach from senior leadership [3]. 
The industry has begun to change with more and more projects making use of new software, mobile devices, the internet and sensors. The first steps came in the 1970s with Computer-Aided Design (CAD). In 2002, Autodesk released a paper entitled "Building Information Modelling" [4] and BIM has been central to technological change in the industry since.

\subsection{BIM: A Step Toward Change}

At its core, BIM is a standardised digital representation of a built asset (such as a building or bridge). This contains data and information linking to spatial relationships, geographic information and properties of building components (e.g. the materials used). More importantly, BIM is a process of standardisation, sharing structured data and managing all the data associated with a building from conception to demolition. With the model at the centre, the aim is for all parties to collaborate on the same rich pool of data but this has not yet been fully achieved.

The adoption of BIM can result in a significant reduction in costs over the lifecycle of a project by detecting issues (such as clashes) early on. Combined with reduced completion times, improved buildings and better safety, the case for adopting BIM is overwhelming. BIM is gaining traction across the world and has been mandatory for all public-sector projects in the UK since April 2016 [5]. Currently, the preconstruction stages widely adopt BIM but it is used progressively less in the later stages of the lifecycle [6]. Fulfilling the potential of BIM for collaboration and data exploitation will require the leveraging of new technology.

\section{A Computing Paradigm Shift for BIM}

BIM has opened the AEC domain to the possibilities of technological change but the scale of the data, required integration of autonomously orchestrated processes, need for seamless collaborative networking and required data intelligence demand new solutions. Contemporary technologies which complement each other and work together can help bring about the required fundamental step-change in the AEC industry. Those that embrace technological change can reap the benefits and become more competitive, as has happened in many other industries.

\subsection{Big Data and Big Data Analytics (BDA)}

Big data can be defined as "High volume, high velocity, and/or high variety information that requires new forms of processing to enable enhanced decision making, insight discovery and process optimization." [7]. Increasing the volume, velocity or variety (the so-called 3Vs of Big Data) increases the data complexity. The $3 \mathrm{Vs}$ are relevant to BIM as follows: 
- Volume - the size of BIM models and the data associated with them (including sensor data) is gradually increasing. In addition, there is an increasing need to look at multiple buildings, the associated infrastructure and their effect on each other.

- Variety - the many formats used in BIM applications include RVT (Revit), IFC, MS formats, sensor data, video, images etc. [6]. The data can be structured, semi-structured or unstructured.

- Velocity - data can be streamed continuously from sensors, building management systems (BMS), etc.

BDA includes techniques from interrelated fields including data mining, machine learning, and artificial intelligence [6]. It is used to discover patterns, relationships and dependencies in the data. These are used to get insights and make predictions. Working with Big Data requires a change of mindset [8]. Vast amounts of data (ideally all of it) are analysed $-\mathrm{a}$ large amount of messy data is considered better than a small amount of exact data and the analysis is more probabilistic than precise. The questions we want to ask sometimes only emerge when we collect and work with all the data. The value of disparate data can exceed its primary purpose when it is combined. Correlations surface from the data instead of requiring a hunch or hypothesis in advance and their existence is more important than their cause. Bilal et al. presented many opportunities for Big Data in the construction industry [6] but its use is still in its infancy.

\subsection{The Internet of Things (IoT)}

The IoT consists of sensors and other devices which send and receive live data via the Internet or other networks. Connecting any asset, machine, system or site to the Internet has an almost limitless range of BIM uses throughout the lifecycle. These include measuring and enhancing facility performance, automation and control, improving safety, energy management, optimising inventories and security [1]. The IoT could save $\$ 1$ trillion dollars a year in maintenance, services and consumables by 2022 [9]. Each IoT solution can require hundreds or thousands of sensors creating a continuous stream of varied data. The IoT and Big Data complement each other with the former providing a rich source of data to be analysed by the latter.

\subsection{Cloud Computing}

Cloud computing is the delivery of computing services over the Internet (i.e. "the cloud"). Most people are familiar with the delivery of cloud storage but servers, databases, networking, analytics, high performance computing and more can also be delivered. This approach frees people from traditional ways of thinking about computing. It brings benefits including real-time 
collaboration, lower cost, elasticity, speed and global scale. Productivity, performance and reliability can all be enhanced [10].

Cloud computing is already starting to be used for BIM. Examples include energy management, stakeholder coordination, structural analysis and the integration of management data [6]. Chuang et al. are utilising Cloud Computing to develop a system for BIM visualisation and manipulation through the web [11].

\section{A Collaborative Unified Computing Platform for BIM}

The proposed platform will combine software, BIM model data and IoT data. The data will be stored in SQL and NoSQL databases, forming a Knowledge Base to be used by the software. BDA can be used to provide insights from the vast amounts of available data, including the real-time analytics of streamed data. A relationship can be maintained between the BIM models and associated IoT devices throughout the lifecycle.

The cloud-based platform will be scalable to multiple built assets all the way up to entire smart cities. A greater amount of data will result in greater potential for BDA to find insights to be exploited in real-time or whenever required. Each project can be stored in the system and analysed. This will provide insights and improvements for future projects and so on.

\subsection{BIM Platform Components and Architecture}

The main components of the BIM Platform are the IFC Engine, Knowledge Base, IoT Hub and Big Data Analytics Engine. Data enters the BIM Platform from BIM Models and IoT Devices. The BIM Platform will allow collaboration between all parties in the BIM process.

The Industry Foundation Classes (IFC) specification is an open and neutral data format used widely throughout BIM applications. IFC files facilitate the sharing process for a better qualification and validation of data [12]. IFC data enters the IFC Engine from BIM Models. A major problem to be overcome by the BIM Platform is that each discipline (architecture, structural engineering, contractors, suppliers etc.) has a separate distinct model. The IFC Engine assembles the distinct models into a federated model which can be stored in the Knowledge Base.

IoT Devices can be connected, managed and monitored by means of an IoT Hub. Data that comes from BIM Models (such as spatial and component data) can provide a framework for the organisation and analysis of IoT data in a way that is useful for the operation of the building [13]. Information coming from apparently unrelated systems can provide valuable, unique and 
actionable insights using BDA. Stream Analytics will be used to provide useful insights from live IoT data in real-time.

Federation combined with IoT data results in a data-rich but extremely complex model. This demands a high performance and smart computing framework. This framework must be able to process data and integrate analysis processes to provide proactive and holistic decision making to benefit all stakeholders. The federated models and IoT data can be analysed using a Big Data Analytics Engine to find insights and new uses for the data. Big Data (federated models, traffic congestion, energy consumption, pressure readings from a bridge etc.) can be collected and subjected to advanced analytics to optimise decision-making and boost operational efficiency. Collaboration between stakeholders and between projects increases the data available and the potential for even deeper insights.

All interactions between the components of the BIM Platform are bidirectional. Therefore, for example, data from the IoT Hub can be stored in the Knowledge Base, analysed by the Big Data Analytics Engine and the results fed back to the IoT Hub (and ultimately the IoT Devices themselves) via the Knowledge Base. Data can flow throughout with great flexibility and the results of analysis can be used for diverse applications. The autonomous and self-managing tools work together dynamically. The BIM Platform will be intelligent and implement tools depending on requirements.

Figure 1 illustrates the conceptual architecture of the BIM Platform. The key features are as follows:

- The external inputs (below the BIM Platform) come from IoT Devices and BIM Models.

- $\quad$ The IoT Hub and IFC Engine can exchange data with IoT Devices and BIM Models respectively.

- IoT data captured by the IoT Hub can be analysed in real-time using Stream Analytics or stored in the Knowledge Base for later analysis by the Big Data Analytics Engine.

- The components of the BIM Platform itself are in the cloud.

- The App elements represent compatible third-party applications and applications developed specifically for the BIM Platform. These connect to the IoT Hub, IFC Engine or Big Data Analytics Engine depending on their purpose.

- Data from all elements is stored in databases (SQL or NoSQL) which together constitute the BIM Platform's Knowledge Base. 


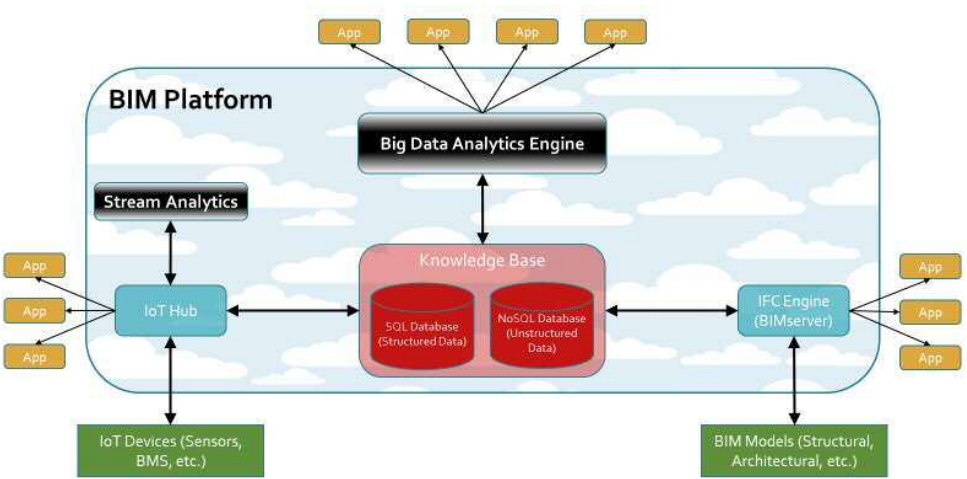

Fig. 1. Architecture of the BIM Platform.

The platform will not have a rigid one-size-fits-all architecture. Each part will have several possible outcomes depending on what is required. The system intelligently determines the best approach based on the advantages and disadvantages of each possibility. Some problems will not require analysing streamed data for example. Each problem will only use a subset of the functionality available. The use of the platform can be expanded as further insights are required.

\subsection{Methodology and Implementation}

The BIM Platform will be hosted using a combination of on-site hardware and Microsoft Azure. The advantages of using on-site hardware include privacy, control and no usage charges. The on-site hardware used includes 8 Intel i7 computers each with 4 cores, 64GB RAM and 4TB of storage. Microsoft Azure is a cloud computing service that can be used for deploying and managing applications, services and additional virtual machines if required. Azure is highly scalable and charges apply for how many resources are used although this project has been awarded an Azure For Research grant to cover the costs. Azure has been used for its power, convenience and flexibility but open-source alternatives to the services used will also be developed in future.

The IFC Engine of the platform is based on BIMserver which runs on an on-site virtual machine. BIMserver adheres to open BIM standards and is an open framework that provides a strong base to build specific BIM applications on top of. BIMserver has an open interface and can be used with any of the various vendors' BIM models. It also has an open API, allowing any applications outside the BIM Platform (e.g. IFC Viewer, BIMserver GUI and IFC Parser) to interact with the models.

The IoT Hub component of the BIM Platform uses Azure IoT Hub for its scalability, multiple communication options, extensive device libraries and 
seamless integration with Azure Stream Analytics to enable powerful realtime analytics. For the purpose of this research, thousands of IoT devices will be emulated using SQL Server and Enzo Unified [14] (both running on on-site virtual machines). Enzo Unified abstracts the underlying cloud APIs, allowing native SQL commands to be executed against the Azure IoT Hub. Apps to connect to outside of the BIM Platform include Device Monitoring, Configuration Management and Content Distribution.

The Knowledge Base uses both SQL and NoSQL databases to store data onsite. BIM model servers generally map IFC entities into their internal structure one-to-one. Because of the inherently complex structure of the IFC schema, this means many tables, one for each IFC entity. In IFC4 the numbers grew to 766 entities and 327 types [15]. This imposes a heavy burden on the relational (SQL) model when the amount of data becomes "big". However, IFC should still be the first choice since it is the main standard supported by major BIM tools. New approaches will be needed to allow fast and efficient queries and analysis. A tweaked schema can flatten the IFC hierarchy into less tables and reduce the number of steps required to access information.

It is beneficial to integrate multiple data sources to facilitate deeper BDA on a combined data set. The structured IFC data of the BIM models themselves will be stored and analysed using SQL databases. There is also an increasing amount of unstructured data [16] associated with the BIM models such as photos, videos, audio, websites, documents (PowerPoint, PDF), scanned documents etc.. The use of NoSQL databases is suitable for a Big Data platform such as this. NoSQL is highly scalable and capable of storing, processing, and managing huge amounts of unstructured data. Combining this with a continuous stream of IoT data results in a large amount of data to be analysed using BDA. The results of these analyses can be used by applications immediately or combined with the original data.

The Big Data Analytics Engine component of the BIM Platform uses Azure HDInsight. HDInsight is a service that deploys and provisions fully managed Apache Hadoop clusters. It provides a software framework for BDA with the analysis and reports being stored in the Knowledge Base. Apps outside of the BIM Platform that can be connected to the Big Data Analytics Engine include Dashboard Tools and Visualisation Tools.

Validation of the BIM Platform will include testing the data from real IoT devices instead of just emulated ones and testing that the integrity of federated BIM Models is maintained upon retrieval from the Knowledge Base after manipulation. Traditional techniques are not viable when validating Big Data and the results of BDA [17]. Autonomous, self-learning Big Data integrity validation and reconciliation tools (e.g. DataBuck) will be used. The accuracy and completeness of the data when moving throughout the BIM Platform will be rigorously validated and checked by 
an expert. Real life case studies will be published in the near future to present how the BIM Platform works in practice.

\section{BIM Platform Uses}

Given the flexible nature of the BIM Platform and the diverse range of tools available, the possible uses are extensive. Some examples include:

- Increased Model Scope - The gradual increase in the size and variety of the contents of BIM models will restrict the capabilities of traditional BIM based storage and processing systems. Up until recently, BIM was envisaged to contain data from the construction industry only. However, the emergence of other linked building data has changed this perception [6]. There is also a far greater need for collaboration. The BIM Platform supports the increase in scope by being scalable in terms of processing power, memory and storage.

- Streamed Data Analysis - The huge amount of data that will increasingly be generated by buildings and infrastructure means they are not just products but providers of services [18]. Rather than the data being archived or just used for its immediate purpose, the BIM Platform enables the analysis of all accumulated data using BDA and also real-time analytics of the data stream using Stream Analytics. For example, sensor data could be analysed to check for the deterioration of a bridge, allowing predictive maintenance [1] and the detection of problems in advance. This not only offers obvious safety benefits but can also help to reduce costs. Parts can be replaced when required instead of at set intervals based on a worst-case scenario.

- Real-Time BIM Model Collaboration - The stakeholders involved in a project can collaborate throughout the BIM process to reduce costs, increase efficiency and address problems early on. Architectural, Structural and MEP (Mechanical Engineering \& Plumbing) models are currently worked on separately and clashes and other issues are only detected at a late stage causing delays or even problems during construction. Using the BIM Platform, they can be worked on simultaneously. IoT data collected during the operation stage of a building can be analysed using BDA and fed back to the stakeholders to make any required changes or to develop improvements in future buildings. The BIM Platform also facilitates collaboration between stakeholders by allowing them to access the same data and share files. This pooled data can be harnessed by BDA to find hidden patterns and deeper insights for the greater good. 
- Smart Cities - The BIM platform allows multiple building and infrastructure projects to be considered simultaneously. Data (including Geographic Information System (or GIS) and IoT data) from whole districts or even entire Smart Cities can be analysed together for insights into urban planning, weather, traffic, fire, etc. BDA and powerful hardware are essential for the consideration of an entire city [19].

- Generative Design - Many building designs can be generated automatically from specified design objectives including functional requirements, material types, energy use and financial goals [6]. Real-time Generative Design is facilitated by parallelised algorithms, BDA and the collaborative aspects of the BIM Platform. Figure 2 illustrates some examples of designs generated by Generative Design [20].
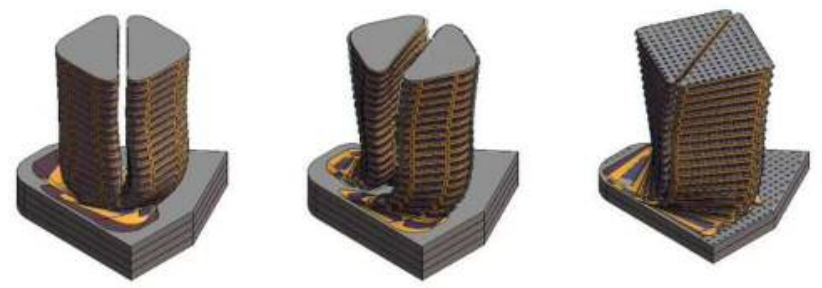

Fig. 2. Examples of designs generated using Generative Design.

\section{Conclusion}

The data traditionally associated with the construction industry will increasingly pale into insignificance compared with the huge amounts of data from multiple sources now beginning to be seen. This data increases exponentially as we move from considering individual buildings to associated infrastructure, districts and even entire smart cities.

Architects, Engineers, operators and owners of built assets will all want to harness this information to generate insight that they can use to become more efficient, save money and make peoples' lives better. This article has explored unifying Cloud Computing, BDA, the IoT and BIM to bring this about. These technologies will play an important role in the future of the AEC industry.

This article has described a BIM Platform which is Big Data enabled, has an IFC compliant BIM engine and an IoT Hub for handling IoT data. The platform is hosted in the cloud to enable collaboration and the linking of BIM models with other sources. There is a wide range of uses for the BIM 
Platform including Increased Model Scope, Streamed Data Analysis, BIM Model Collaboration, Smart Cities and Generative Design.

There will be ongoing hurdles to overcome including security, privacy and changing well established processes. There will be compatibility issues between the disparate technologies used in the BIM Platform and by stakeholders so new software will need to be developed. However, these issues have been common in other fields and have been overcome as change was embraced. In the AEC domain, there is still a lingering resistance to change and an understandable suspicion of sharing data amongst stakeholders. With determination, this can be overcome as the long-term advantages become irresistible.

There is a great deal of scope for future work. The BIM Platform forms a foundation for other apps to be connected to. The Apps referred to in this article are a subset of what is possible and Apps will be developed on an ongoing basis. The BIM Platform will connect to compatible third party Apps or new Apps will be developed as required. In future the functionality of the BIM Platform will be expanded to integrate additional data sources into the Knowledge Base. For example, the integration of social media data could lead to insights into what people want from buildings, deciding where new buildings should be located and learning about attitudes to buildings that already exist.

The potential benefits of the BIM Platform include increased efficiency, greater collaboration, new insights from data and reduced costs. But, more generally, it is hoped that it will move forward and accelerate the changes that the industry has started to make. From CAD to BIM and now to a Collaborative Unified Computing Platform for BIM. Unifying BIM with Cloud Computing, BDA and the IoT can help bring about the step-change the AEC domain needs.

\section{References}

1. World Economic Forum: What's the future of the construction industry? (2016).

https://www.weforum.org/agenda/2016/04/building-in-the-fourthindustrial-revolution/. Accessed March 2017

2. A. Weippert and S. L. Kajewski, "AEC industry culture: A need for change,” CIB World Build. Congr. 2004 Build. Futur., pp. 1-10, 2004.

3. D. Migilinskas, V. Popov, V. Juocevicius, and L. Ustinovichius, "The benefits, obstacles and problems of practical bim implementation," Procedia Eng., vol. 57, pp. 767-774, 2013.

4. Autodesk Revit: Building Information Modelling For Sustainable Design (2005). 
http://images.autodesk.com/latin am main/files/bim for sustainable design_oct08.pdf. Accessed March 2017

5. Designing Buildings Wiki: Federated building information model (2016).

https://www.designingbuildings.co.uk/wiki/Federated_building_infor mation_model. Accessed March 2017

6. M. Bilal et al., "Big Data in the construction industry: A review of present status, opportunities, and future trends," Adv. Eng. Informatics, vol. 30, pp. 500-521, 2016.

7. Gartner: Big Data (2017). http://www.gartner.com/it-glossary/bigdata. Accessed March 2017

8. Mayer-Schönberger, V: Big data : a revolution that will transform how we live, work and think (2013).

9. Information Management: Top 10 Predictions for IT in 2017 and Beyond (2016). $\quad \underline{\text { http://www.information- }}$ management.com/gallery/oct-top-reader-pick-top-10-predictions-forit-in-2017-and-beyond-10030035-1.html. Accessed March 2017

10. Microsoft Azure: What is cloud computing? (2017). https://azure.microsoft.com/en-gb/overview/what-is-cloudcomputing/. Accessed March 2017

11. T.-H. Chuang, B.-C. Lee, and I.-C. Wu, "Applying Cloud Computing Technology To BIM Visualization And Manipulation.” (2011)

12. R. Vanlande, C. Nicolle, and C. Cruz, "IFC and building lifecycle management," Autom. Constr., vol. 18, no. 1, pp. 70-78, 2008.

13. FM Systems: Does BIM have a role in the Internet of Things? (2016). https://fmsystems.com/blog/does-bim-have-a-role-in-the-internet-ofthings. Accessed March 2017

14. Enzo Unified: Edge Computing Platform (2017). http://www.enzounified.com/. Accessed March 2017

15. W. Solihin and C. Eastman, "A Simplified BIM Model Server on a Big Data Platform." 2016.

16. Conject: Are asset owners and the construction industry really ready for 'Big Data'? (2017). http://www.conjectblog.com/are-assetowners-and-the-construction-industry-really-ready-for-big-data/. Accessed March 2017

17. FirstEigen: Big Data Quality, Integrity Validation, Reconciliation Tool (2017). http://firsteigen.com/databuck/. Accessed March 2017

18. D. Pasini, S. Mastrolembo Ventura, S. Rinaldi, P. Bellagente, A. Flammini, and A. L. C. Ciribini, "Exploiting Internet of Things and building information modeling framework for management of cognitive buildings," 2016 IEEE Int. Smart Cities Conf., vol. 40545387, no. 40545387, pp. 1-6, 2016.

19. F. R. Correa, "Is BIM Big Enough to Take Advantage of Big Data Analytics??," 2015.

20. Autodesk: BIM and the Net-Zero Building (2015) http://sustainability.autodesk.com/blog/bim-net-zero-building/. Accessed March 2017 